\title{
Stürzt der Tiers payant die Ärzte ins Verderben?
}

\author{
Standespolitisch wird ein Wechsel zum Tiers payant als Bedrohung der ärzteeigenen \\ Datenbasis und des Taxpunktwertes bekämpft. Aufgrund des Strukturwandels und \\ im Zuge der Einführung der elektronischen Krankengeschichte bietet er sich jetzt aber \\ als wesentlich einfacherer und kostengünstigerer Abrechnungsmodus an.
}

Franz Marty

Ein ausführlicher Kommentar der FMH zu diesem Artikel folgt in einer der nächsten Ausgaben.
1 Gähler E, Marty I, Schutz K. Die FMH für den Tiers garant. Schweiz Ärztezeitung. 2014;95(5):147-9.

2 Wiedersheim P. Was für den Tiers Garant spricht: KKA und $\mathrm{K}-\mathrm{OCH}$ befürworten wie die FMH klar den Tiers Garant! HKO-Bulletin Nr. 5 (PDF, $1002 \mathrm{~KB}$ ).

3 Die FMH für den Tiers garant Positionspapier der FMH.

Korrespondenz: Franz Marty

Arzt für Allgemeinmedizin

Gürtelstrasse 46

CH-7000 Chur

franz.marty[at]hin.ch
In der ambulanten Medizin sah der Gesetzgeber den Tiers garant vor. Die Spitäler und die Apotheken rechnen im Tiers payant $a b$, ein grosser Teil der niedergelassenen Ärzte im deutlich aufwendigeren Tiers garant. Standespolitisch wird ein Wechsel zum Tiers payant als Bedrohung der ärzteeigenen Datenbasis und des Taxpunkt-Wertes bekämpft. Dieser ärztliche Alleingang im Tiers garant lässt sich in Zukunft allerdings kaum halten. Der folgende Text entstand aus einer internen Standortbestimmung (Nov. 2013) eines ärzteeigenen medizinischen Zentrums und nimmt Bezug auf die offizielle Position «Die FMH für den Tiers garant» in der SÄZ 5/2014 [1].

\section{Einleitung}

Die Debatte, ob im Tiers garant (TG) oder im Tiers payant (TP) abgerechnet werden soll, sorgt standespolitisch seit langem immer wieder für Kontroversen. Die Diskussionen innerhalb der Ärzteschaft werden zunehmend zu einem Lackmustest für die Verbandstreue [2], die FMH erinnert in der Ärztezeitung an die offizielle Position [1, 3]. Als Haupt-Argumente gegen einen Wechsel in den TP werden die Bedrohung des Taxpunkt-Wertes und die Schwächung der ärzteeigenen Datenbasis aufgeführt.

Andere wichtige Aspekte haben auf die Wahl des Abrechnungsmodus aber ebenso starken Einfluss: Der Bedarf des Patienten, der Aufwand der Rechnungsstellung für den Leistungserbringer, die Kosten des Mahnwesens, die Gefahr einer Marginalisierung administrativ aufwendiger Patienten oder die weitere Entwicklung im Rahmen von eHealth.

Viele Patienten, aber auch die Grosspraxen stossen mit der Abrechnung im TG an die Grenzen. Für viele Patienten sind die Arztrechnungen eine grosse finanzielle Belastung, die Umtriebe mit den Rückforderungsbelegen eine mühsame Angelegenheit. Bei den Praxen, insbesondere Grosspraxen, führen das Mahnwesen und die Verwaltung der Abtretungserklärungen (direkte Abrechnung mit der Krankenkasse im TG) zu einem nicht mehr vertretbaren administrativen Aufwand. Zudem sind die Aussichten einer Vereinfachung der Fakturierung im TG (elektronische Rechnungsstellung, eHealth) nicht rosig.

Die Standortbestimmung gliedert sich in die drei Teile: «Soll-Zustand» aus der Zeit der KVG-Revision / Einführung des TARMED, die «aktuelle Situation» und die «Aussicht auf die weitere Entwicklung».

\section{Le Tiers payant cause-t-il la perte des médecins?}

Depuis de nombreuses années, les débats autour du mode de facturation Tiers garant (TG) vs Tiers payant (TP) suscitent régulièrement la controverse au sein de la profession. La FMH invite ses membres à facturer leurs prestations selon le modèle du TG et en appelle à la solidarité. Selon elle, un passage au système du TP occasionnerait notamment une diminution de la valeur du point tarifaire et un affaiblissement de la base de données du corps médical.

Or l'argumentation de la position officielle repose sur des conditions qui prévalaient à la fin des années 90 au moment de l'introduction du TARMED. Elle ne correspond plus à la réalité actuelle et empêche toute vision d'avenir. Coûteux et peu apprécié des patients, le TG ne s'est pas imposé dans le secteur ambulatoire (pharmacies et hôpitaux), et sera difficile à adapter à la cybersanté. En persistant à l'utiliser, les médecins risquent de finir par cesser toute collaboration avec les centres de confiance et par céder une part de leur propre base de données à MediData. En outre, pour les patients en difficulté financière, l'accès au système de santé deviendra plus difficile. Si le TG a permis au corps médical de créer des centres de confiance (réseaux) ainsi que NewIndex et de mettre sur pied sa propre base de données, un passage au TP ne compromettrait pas ces nouvelles structures. Le mode de facturation devrait pouvoir être choisi librement.

\section{Soll-Zustand}

\section{Gesetzliche Grundlage}

Schon 1991 empfahl der Bundesrat in der «Botschaft über die Revision der Krankenversicherung» den TG: «Die Vorlage legt Wert darauf, die Verantwortung und das Kostenbewusstsein der Versicherten zu stärken. Sie 
stellt daher das System des Tiers garant in den Vordergrund.» [4] Das Parlament entschied sich in der Revision des Krankenversicherungsgesetzes (KVG) 1994 dann auch für den Tiers garant im ambulanten Bereich. Der Artikel 42 im KVG aus dem Jahre 1994 lautet [5]:

${ }^{1}$ Haben Versicherer und Leistungserbringer nichts anderes vereinbart, so schulden die Versicherten den Leistungserbringern die Vergütung der Leistung. Die Versicherten haben in diesem Fall gegenüber dem Versicherer einen Anspruch auf Rückerstattung (System des Tiers garant). In Abweichung von Artikel 22 Absatz 1 ATSG kann dieser Anspruch dem Leistungserbringer abgetreten werden. ${ }^{2}$ Versicherer und Leistungserbringer können vereinbaren, dass der Versicherer die Vergütung schuldet (System des Tiers payant). Im Falle der stationären Behandlung schuldet der Versicherer, in Abweichung von Absatz 1, den auf ihn entfallenden Anteil an der Vergütung.

Der Gesetzgeber setzte als «Soll-Einstellung» also den TG, liess es den Parteien allerdings frei, sich für den TP zu entscheiden.

\section{Die standespolitische Position}

Die heutige Position der FMH ist eine standespolitische und stammt aus dem gesundheitspolitischen Kontext und der Versorgungssituation Ende der

Articles interactifs

Vous souhaitez commenter cet article? II vous suffit pour cela d'utiliser la fonction «Ajouter un commentaire» dans la version en ligne. Vous pouvez également consulter les remarques de vos confrères sous: www.bullmed.ch/ numero-actuel/ articles-interactifs/
4 Protokolle Bundesverwaltung www.amtsdruckschriften. bar.admin.ch/viewOrigDoc do?id=10052098 Seite 170

5 Gesetzessammlung Bund www.admin.ch/opc/de/ classified-compilation/ 19940073/index.html

6 Website NewIndex AG - Your Medical Data Insights Daten und Dienstleistungen.

7 Laffranchi R, Stoffel U. Braucht die Ärzteschaft überhaupt eigene Daten? Schweiz Ärztezeitung. 2013;94(43):1610-2.

8 Persönliche Mitteilung eines Software-Supporters. 90er Jahre. Sie folgte dem Willen des Gesetzgebers. In vielen Kantonen bedeutete dies einen Wechsel vom damals üblichen TP zum TG.

Der TG lag in zweifachem Interesse der Ärzteschaft: Er erlaubte erstmals den Aufbau einer eigenen Datenbasis. Zudem erhoffte man sich mit dem TG einen guten Taxpunkt-Wert im Übergang von den kantonalen Tarifen zum TARMED. Dieser hatte ja kostenneutral zu erfolgen. Was die Versorgungssituation betrifft, waren die Patienten viel weniger mobil, die Praxislandschaft bestand, bis auf Ausnahmen, aus Einzelpraxen mit fester Klientel. Es bestand auch ein Wunsch nach Transparenz, der Patient sollte die Arztrechnung einsehen können.

\section{Stand der Dinge}

Im Rückblick auf 20 Jahre revidiertes KVG lässt sich Folgendes festhalten:

\section{Der TG hat sich nicht durchgesetzt}

Im ambulanten Bereich rechnen zwar viele Ärzte im TG ab, insgesamt hat er sich aber nicht durchgesetzt: Die Spitäler und Apotheken, die beiden andern grossen Leistungserbringer-Gruppen im ambulanten Sektor, rechnen nicht mit dem Versicherten, sondern mit den Krankenkassen ab.

\section{Die Trustcenter und Ärztenetzwerke sind stabile Institutionen}

Der TG ermöglichte, via Einrichtung regionaler Trustcenter, den Aufbau einer ärzteeigenen Datenbasis (New Index). Die Trustcenter (TC) entwickelten sich zu sta- bilen Ärztenetzen und Dienstleistungsunternehmen, NewIndex ist als ärzteeigener Datenpool [6] im Gesundheitssystem anerkannt und für die Ärzte nach innen und aussen von grossem Nutzen [7].

Die von Ärzteseite (NewIndex, TC) zur Einführung des TARMED entwickelte elektronische Übermittlung der Rechnungen hat sich nicht erwartungsgemäss entwickelt. Die Kassen holten die für sie kostenpflichtigen elektronischen Rechnungen bei den TC nicht ab. Im Gegenzug stellten sie nun ihren eigenen, für den TP konzipierten MediData-Kanal kostenlos (anstelle von 50 Rappen pro Rechnung) zur Verfügung. Und sie forderten von den TC ebenfalls eine kostenlose Lieferung der dort gesammelten Arztrechnungen.

Der Stand der Dinge ist, dass Ärzte heute via MediData kostenlos elektronisch abrechnen können und Ärzte, die via TC abrechnen, zwar jährlich, zwecks Datensammlung, Millionen von elektronischen Rechnungen an die TC schicken, eine medienbruchfreie Übernahme der Rechnungen durch die Versicherer aber bis heute nicht zustande kam. Parallel zum elektronischen Versand an die TC müssen die Ärzte ihren Patienten weiterhin die Rechnung auf Papier zustellen - und die Versicherer scannen die von den Patienten an sie weitergeleiteten Rechnungen zur weiteren administrativen Verarbeitung ein!

\section{Rascher Strukturwandel}

Der Wechsel in den TG anlässlich der Einführung des TARMED wurde von den Ärzten grossmehrheitlich akzeptiert und von den meisten solidarisch befolgt. Der rasante Strukturwandel der letzten 10 Jahre (weniger Praxen, dafür grosse Einheiten, Verschlechterung der Zahlungsmoral) setzte den TG unter Druck. Viele Ärzte wechselten in den TP, und viele ziehen heute einen Wechsel in Betracht. Neu eröffnende Praxen rechnen, bis auf Ausnahmen, im TP ab [8].

\section{Die weitere Entwicklung}

Der äussere Druck wird weiterhin die Wahl des Abrechnungsmodus steuern.

\section{Tiers garant}

Der TG wird es in Zukunft aller Voraussicht nach schwer haben. Er zeigt einige gravierende Nachteile:

- Leute mit kleinem Geldbeutel, wie AHV-Bezüger, junge Familien, Asylanten, Behinderte, Suchtkranke und Patienten mit schlechter Zahlungsmoral, aber auch polymorbide ältere Patienten in sozial guten Verhältnissen, sind im TG finanziell oft überfordert.

- Es zeichnet sich so die Gefahr ab, dass ein beträchtlicher Teil der Bevölkerung in Zukunft ausgegrenzt wird oder nur mit Mühe eine adäquate medizinische Betreuung finden wird.

- In den Kantonen mit freier Wahl des Medikamentenbezuges (Selbstdispensation) verschärft sich dieses Problem noch: Im TG beziehen diese Patienten zu ihrer Entlastung die Medikamente des- 
halb lieber in der Apotheke. Die Apotheke rechnet im TP ab.

- Der Aufwand für das Mahnwesen im TG wird immer bedeutend grösser sein als im TP.

- Je nach Praxisklientel kann sich der Arzt nur mit Abtretungserklärungen gegen grosse Debitorenverluste schützen (nach KVG Absatz 2, siehe oben). Die Versicherer fordern nun zunehmend, dass mit jeder neuen Rechnung eine neuausgestellte Abtretungserklärung versandt werden muss. Für Grosspraxen ergibt sich damit ein nicht mehr zu rechtfertigender administrativer Aufwand.

- Die Einbindung des TG in den elektronischen Verarbeitungsprozess (eHealth) ist schwierig, und in Zukunft vermutlich das grösste Hindernis für den Erfolg des TG.
- Gefährdung des Taxpunkt-Wertes: Bei der Abrechnung im TP erhöht sich das Rechnungsvolumen an die Krankenkasse, da im TG Arztrechnungen, welche die Jahresfranchise nicht übersteigen, oft nicht an die Krankenkassen gesandt werden. Da LeiKoV [10] das Rechnungsvolumen als wichtiges Kriterium für die Bestimmung des TP-Wertes verwendet, kann der Wechsel in den TP einen Einfluss auf den Taxpunkt-Wert haben (das Rechnungsvolumen soll im TP um 750 Millionen Franken anwachsen [1]).

Die Relevanz dieser Befürchtung ist allerdings nicht klar: In der Ostschweiz rechnen heute die meisten Grosspraxen und viele Einzelpraxen im TP ab, bisher ohne Wirkung auf den TaxpunktWert und ohne Rückmeldungen auf ein stark gewachsenes Kostenvolumen.

\section{Mit Beharren der Ärzte auf dem Tiers garant besteht die Gefahr, finanz- schwachen Patienten den Zugang zum Gesundheitssystem zu erschweren.}

\section{Tiers payant}

Der TP hat demgegenüber für die Zukunft die deutlich besseren Karten:

- Der Hauptvorteil des TP liegt in einer markanten Verminderung des Debitorenaufwandes und nicht, wie in der offiziellen Position argumentiert, in der Eindämmung der Debitorenverluste.

- Der TP lässt sich wesentlich besser in den eHealthWorkflow integrieren. Die TrustX-Schnittstelle zur elektronischen Abrechnung ist in der Praxissoftware aller Anbieter integriert. Die elektronischen Rechnungen liegen in den Trustcenters schon vor.

- Der TP stellt die Ärzte den andern Rechnungsstellern im ambulanten Bereich (Spital ambulant, Apotheken) gleich.

- Der TP kann die ärztliche Datenbasis stärken, sofern über die TCs abgerechnet wird [9], d.h. diese sich dem TP nicht verschliessen.

- Der TP ermöglicht auch in Zukunft allen Bevölkerungsgruppen einen fairen Zugang zur Primärversorgung.

Die Argumente gegen den TP überzeugen zu wenig.

- Rechnungskontrolle durch den Patienten: Die Rechnungs-Aufstellung ist für die meisten (TARMEDunkundigen) Patienten nicht verständlich. Und es besteht im TP weiterhin die Möglichkeit, dies wie bisher zu handhaben.

Erich Honegger. HKOInformation Nr. 6. www.hk-o.ch/documents/ Bulletin6.pdf

10 Stoffel U. Die Leistungs- und Kostenvereinbarung (LeiKoV) als Prinzip. www.primary-care.ch/docs/ primarycare/archiv/ defr/2005/2005-40/ 2005-40-472.PDF
Es bleibt in dieser Sache noch anzufügen, dass, aus einer systemischen Sicht, Referenzgrössen zur Bestimmung des Taxpunkt-Wertes nicht vom Rechnungsmodus abhängen sollten. Solche sachfremden Abhängigkeiten sind gefährlich und können sinnvolle weitere Entwicklungen hemmen.

\section{Schlussfolgerungen}

Es lässt sich festhalten, dass 20 Jahre nach Einführung des KVG aus der Absicht des Gesetzgebers nicht viel geworden ist: Der TG wurde nur von den frei praktizierenden Ärzten befolgt, er hat sich insgesamt (Spitäler ambulant und Apotheken) nicht durchgesetzt. Für die Ärzte war der Wechsel in den TG ohne Zweifel von grossem Nutzen: Er wirkte solidaritätsstiftend und erlaubte den Aufbau wertvoller Strukturen (NewIndex, Trustcenters, Ärztenetzwerke). Im Zuge der Einführung der elektronischen Krankengeschichte sowie des rasanten Strukturwandels (Grosspraxen und medizinische Zentren) bietet sich heute der TP als der wesentlich einfachere und kostengünstigere Abrechnungsmodus an.

Mit Beharren der Ärzte auf dem TG besteht die Gefahr, finanzschwachen Patienten den Zugang zum Gesundheitssystem zu erschweren und Kunden der Trustcenters an MediData zu verlieren. Die ärzteeigene Datenbasis ist mit einem Wechsel zum TP nicht gefährdet - es besteht vielmehr die Chance, diese zu erweitern und die Position der TC zu stärken. Diese können so ihren Kunden weiterhin ein «allround-Dienstleistungspaket» anbieten.

Die Wahl des Abrechnungsmodus sollte freigegeben werden. Der Patient soll über den Abrechnungsmodus entscheiden können. 